\title{
A Further Research on the Convergence of Wu-Schaback's Multi-quadric Quasi-Interpolation
}

Yang Zhang, Xue-Zhang Liang, Qiang Li*

School of Mathematics, Jilin University, Changchun, 130012, P.R. China

\begin{abstract}
The paper discusses the error estimate of Wu-Schaback's quasi-interpolant for a wider class of approximated functions (the functions with lower smoothness order). Three cases are considered: a function with a Lipschitz continuous first-order derivative, a continuous function and a Lipschitz continuous function, respectively.
\end{abstract}

Keywords: Quasi-Interpolation; Multi-quadric; Convergence; Error estimate

\section{Introduction}

Quasi-interpolation methods have been used widely in data analysis, and have great values not only in theory but also in many application areas such as medicine, geology, economy and computer science. Multiquadric functions were first proposed by Hardy [1] in 1968, and Franke [2] showed they performed well in many calculations including the numerical experiments. Powell [3], Beatson and Powell [4], and Beatson and Dyn [5] successively proposed a number of quasiinterpolation schemes and discussed the convergence of the schemes. In 1994, Wu and Schaback [6] proposed a useful quasi-interpolation operator $L_{D} f$ and discussed the convergence and shape preserving properties of this operator. In their convergence theorem (theorem A in our paper), they claimed interpolated functions $f(x) \in C^{2}$. Based on these papers, Zhang and $\mathrm{Wu}$ [7], and $\mathrm{Ma}$ and $\mathrm{Wu}$ [8] did further researches. In this paper, we discuss the convergence of operator $L_{D} f$ for a wider range of approximated functions (namely functions with lower smoothness). To prove the convergence, we use two theorems showed by Beatson and Powell [4], and our method differs from that in [6].

\section{Preparation}

We assume that there are finite scattered points $\left\{x_{j}\right\}_{j=0}^{N}$ in the bounded interval $[a, b]$ as follows:

$$
a=x_{0}<x_{1}<\ldots<x_{N-1}<x_{N}=b,
$$

and the maximum spacing is defined as

$$
h=\max _{1 \leq j \leq N}\left(x_{j}-x_{j-1}\right) .
$$

For $f \in C[a, b]$, we define its norm as

$$
\|f\|_{\infty}=\max _{a \leq x \leq b}|f(x)|,
$$

and its modulus of continuity as

$$
\omega(f, \delta)=\max _{\substack{a \leq x, x+h \leq b \\|h| \leq \delta}}|f(x+h)-f(x)| .
$$

The basis functions used in this paper are

$$
\begin{aligned}
& \phi_{j}(x)=\sqrt{c^{2}+\left(x-x_{j}\right)^{2}}, j=0, \ldots, N, \\
& \psi_{j}(x)=\frac{\phi_{j+1}(x)-\phi_{j}(x)}{2\left(x_{j+1}-x_{j}\right)}-\frac{\phi_{j}(x)-\phi_{j-1}(x)}{2\left(x_{j}-x_{j-1}\right)}, j=1, \ldots N-1,
\end{aligned}
$$

where $c>0$ is a positive shape parameter

In 1994, Wu and Schaback proposed the quasi-interpolation operator $L_{D}$ :

$$
\left(L_{D} f\right)(x)=f_{0} \alpha_{0}(x)+f_{1} \alpha_{1}(x)+\sum_{j=2}^{N-2} f_{j} \psi_{j}(x)+f_{N-1} \alpha_{N-1}(x)+f_{N} \alpha_{N}(x),
$$

where

$$
\begin{aligned}
& \alpha_{0}(\mathrm{x})=\frac{1}{2}+\frac{\phi_{1}(\mathrm{x})-\left(\mathrm{x}-\mathrm{x}_{0}\right)}{2\left(\mathrm{x}_{1}-\mathrm{x}_{0}\right)}, \\
& \alpha_{1}(\mathrm{x})=\frac{\phi_{2}(\mathrm{x})-\phi_{1}(\mathrm{x})}{2\left(\mathrm{x}_{2}-\mathrm{x}_{1}\right)}-\frac{\phi_{1}(\mathrm{x})-\left(\mathrm{x}-\mathrm{x}_{0}\right)}{2\left(\mathrm{x}_{1}-\mathrm{x}_{0}\right)}, \\
& \alpha_{N-1}(\mathrm{x})=\frac{\left(\mathrm{x}_{N}-\mathrm{x}\right)-\phi_{N-1}(\mathrm{x})}{2\left(\mathrm{x}_{N}-\mathrm{x}_{N-1}\right)}-\frac{\phi_{N-1}(\mathrm{x})-\phi_{N-2}(\mathrm{x})}{2\left(\mathrm{x}_{N-1}-\mathrm{x}_{N-2}\right)}, \\
& \alpha_{N}(\mathrm{x})=\frac{1}{2}+\frac{\phi_{N-1}(\mathrm{x})-\left(\mathrm{x}_{N}-\mathrm{x}\right)}{2\left(\mathrm{x}_{N}-\mathrm{x}_{N-1}\right)} .
\end{aligned}
$$

They got the error estimate of this operator as follows:

Theorem A: For $f \in C^{2}[a, b]$ the quasi-interpolant $L_{D} f$ satisfies an error estimate of type

$$
\left\|f-L_{D} f\right\|_{\infty} \leq K_{1} h^{2}+K_{2} c h+K_{3} c^{2} \log h,
$$

where positive constants $K_{1}, K_{2}, K_{3}$ are independent of $h$ and $c$.

In 1992, Beatson and Powell [4] proposed the quasi-interpolation operator $L_{B}$ :

$$
\left(L_{B} f\right)(x)=f\left(x_{0}\right) \beta_{0}(x)+\sum_{j=1}^{N-1} f\left(x_{j}\right) \psi_{j}(x)+f\left(x_{N}\right) \beta_{N}(x), \quad x \in R,
$$
where

$$
\begin{array}{ll}
\beta_{0}(x)=\frac{1}{2}+\frac{\left[\left(x-x_{1}\right)^{2}+c^{2}\right]^{1 / 2}-\left[\left(x-x_{0}\right)^{2}+c^{2}\right]^{1 / 2}}{2\left(x_{1}-x_{0}\right)}, & x \in R, \\
\beta_{N}(x)=\frac{1}{2}-\frac{\left[\left(x-x_{N}\right)^{2}+c^{2}\right]^{1 / 2}-\left[\left(x-x_{N-1}\right)^{2}+c^{2}\right]^{1 / 2}}{2\left(x_{N}-x_{N-1}\right)}, & x \in R .
\end{array}
$$

*Corresponding author: Qiang Li, School of Mathematics, Jilin University, Changchun, 130012, PR China, E-mail: liq@jlu.edu.cn

Received August 25, 2013; Accepted September 23, 2013; Published September 26, 2013

Citation: Zhang Y, Liang XZ, Li Q (2013) A Further Research on the Convergence of Wu-Schaback's Multi-quadric Quasi-Interpolation J Appl Computat Math 2: 138. doi:10.4172/2168-9679.1000138

Copyright: ( 2013 Zhang Y, et al. This is an open-access article distributed under the terms of the Creative Commons Attribution License, which permits unrestricted use, distribution, and reproduction in any medium, provided the original author and source are credited. 
They proved the following result:

Theorem B: In interval $[a, b]$, the error function $\left\{f(x)-\left(L_{B} f\right)(x)\right\}$ satisfies the bound

$$
\left\|f-L_{B} f\right\|_{\infty} \leq(1+c / h) \omega(f, h) .
$$

Meanwhile, in [4] the quasi-interpolation operator $L_{C}$ was defined as follows:

$$
\left(L_{C} f\right)(x)=\left(L_{B} f\right)(x)+f^{\prime}\left(x_{0}\right) \gamma_{0}(\mathrm{x})+f^{\prime}\left(x_{N}\right) \gamma_{N}(\mathrm{x}), \quad \mathrm{x} \in \mathrm{R},
$$

where

$$
\begin{array}{ll}
\gamma_{0}(x)=\frac{1}{2}\left(x-x_{0}\right)-\frac{1}{2}\left[\left(x-x_{0}\right)^{2}+c^{2}\right]^{1 / 2}, & x \in R, \\
\gamma_{N}(x)=\frac{1}{2}\left[\left(x_{N}-x\right)^{2}+c^{2}\right]^{1 / 2}-\frac{1}{2}\left(x_{N}-x\right), & x \in R .
\end{array}
$$

They got the following theorem:

Theorem C: If $f$ has a Lipschitz continuous first-order derivative, then the maximum error of the quasi-interpolant $L_{C} f$ satisfies the bound

$$
\left\|f-L_{C} f\right\|_{\infty} \leq \frac{1}{4} c^{2} \Omega\left[1+2 \log \left(1+\frac{b-a}{c}\right)\right]+\frac{1}{8} h^{2} \Omega,
$$

where $\Omega=e s s \sup \left|f^{\prime \prime}(x)\right|$

$$
a \leq x \leq b
$$

\section{Main Result}

It should be noticed that in Theorem A, Wu and Schaback demanded the approximated function $f \in C^{2}[a, b]$. In this paper, we weaken this condition step by step. Using Theorem B and Theorem C proposed by Beatson and Powell, we get three theorems about convergence estimate for the approximated functions with lower smoothness.

Theorem 1: If $f$ has a Lipschitz continuous first-order derivative, then we can draw the conclusion:

$$
\left\|f-L_{D} f\right\|_{\infty} \leq M c h+\frac{1}{4} c^{2} \Omega\left[1+2 \log \left(1+\frac{b-a}{c}\right)\right]+\frac{1}{8} h^{2} \Omega,
$$

where $M=\underset{a \leq x \leq b}{\operatorname{ess} \sup }\left|f^{(2)}(x)\right|$.

Proof: We notice that quasi-interpolant $L_{D} f$ and $L_{B} f$ have the following relationship:

$$
L_{D} f=L_{B} f+\frac{f\left(x_{1}\right)-f\left(x_{0}\right)}{x_{1}-x_{0}} \gamma_{0}(x)+\frac{f\left(x_{N}\right)-f\left(x_{N-1}\right)}{x_{N}-x_{N-1}} \gamma_{N}(x) .
$$

In [4], Beatson and Powell have showed the relationship between $L_{B} f$ and $L_{C} f$ :

$$
L_{C} f=L_{B} f+f^{\prime}\left(x_{0}\right) \gamma_{0}(x)+f^{\prime}\left(x_{N}\right) \gamma_{N}(x),
$$

where

$$
\begin{aligned}
& \gamma_{0}(\mathrm{x})=\frac{1}{2}\left(\mathrm{x}-\mathrm{x}_{0}\right)-\frac{1}{2} \phi_{0}(\mathrm{x}), \\
& \gamma_{N}(\mathrm{x})=\frac{1}{2} \phi_{N}(\mathrm{x})-\frac{1}{2}\left(\mathrm{x}_{N}-\mathrm{x}\right) .
\end{aligned}
$$

For $x \in[a, b]$, we can easily get the two inequalities:

$$
\left|\gamma_{0}\right| \leq \frac{1}{2} c,\left|\gamma_{N}\right| \leq \frac{1}{2} c \text {. }
$$

Using (1), (2),(3), we can get

$$
\begin{aligned}
\left|L_{D} f-L_{C} f\right| & =\left|\left[\frac{f\left(x_{1}\right)-f\left(x_{0}\right)}{x_{1}-x_{0}}-f^{\prime}\left(x_{0}\right)\right]\right|\left|\gamma_{0}(x)\right|+\left|\left[\frac{f\left(x_{N}\right)-f\left(x_{N-1}\right)}{x_{N}-x_{N-1}}-f^{\prime}\left(x_{N}\right)\right]\right|\left|\gamma_{N}(x)\right| \\
& \leq \frac{1}{2} c\left|f^{\prime}\left(x_{0}+\theta \Delta x_{0}\right)-f^{\prime}\left(x_{0}\right)\right|+\frac{1}{2} c\left|f^{\prime}\left(x_{N}-\theta \Delta x_{N-1}\right)-f^{\prime}\left(x_{N}\right)\right|
\end{aligned}
$$

Further, dued Theorem $C$, we can get

$$
\begin{aligned}
\left\|L_{D} f-f\right\|_{\infty} & \leq\left\|L_{D} f-L_{C} f\right\|_{\infty}+\left\|L_{C} f-f\right\|_{\infty} \\
& \leq M c h+\frac{1}{4} c^{2} \Omega\left[1+2 \log \left(1+\frac{b-a}{c}\right)\right]+\frac{1}{8} h^{2} \Omega . \quad \#
\end{aligned}
$$

Remark 1: Usually we choose $c=O(h)$, then Theorem 1 is basically in accordance with Theorem A.

Further, for the approximated function $f(x)$ with lower smoothness, we can get the following results:

Theorem 2: If is $\mathrm{f}(\mathrm{x})$ Lipschitz continuous in $[\mathrm{a}, \mathrm{b}]$, then

$$
\left\|f-L_{D} f\right\|_{\infty} \leq M c+(1+c / h) \omega(f, h),
$$

where $M=\underset{a \leq x \leq b}{\operatorname{ess} \sup }\left|f^{\prime}(x)\right|$

Proof: Due to (3), it is obvious that

$$
\begin{aligned}
\left|L_{D} f-L_{B} f\right| & =\left|\left[\frac{f\left(x_{1}\right)-f\left(x_{0}\right)}{x_{1}-x_{0}}\right]\right| \gamma_{0}(x)|+|\left[\frac{f\left(x_{N}\right)-f\left(x_{N-1}\right)}{x_{N}-x_{N-1}}\right]\left|\gamma_{N}(x)\right| \\
& \leq M c,
\end{aligned}
$$

Finally, using Theorem B, we have

$$
\begin{gathered}
\left\|L_{D} f-f\right\|_{\infty} \leq\left\|L_{D} f-L_{B} f\right\|_{\infty}+\left\|L_{B} f-f\right\|_{\infty} \\
\leq M c+(1+c / h) \omega(f, h) .
\end{gathered}
$$

Remark 2: Since $f(x)$ is Lipschitz continuous in $[a, b]$ and $M=\operatorname{ess} \sup _{a \leq x \leq b}\left|f^{\prime}(\mathrm{x})\right|$ we have $\omega(f, h)=\max _{\substack{a \leq x_{1}, x_{2} \leq b \\\left|x_{1}-x_{2}\right| \leq h}}\left|f\left(x_{1}\right)-f\left(x_{2}\right)\right| \leq M h$.

Then Theorem 2 can be rewrote as:

If $f(x)$ is Lipschitz continuous in interval $[a, b]$, then

$\left\|f-L_{D} f\right\|_{\infty} \leq M c+(1+c / h) M h=M(h+2 c)$,

where $M=\underset{a \leq x \leq b}{\operatorname{ess} \sup }\left|f^{\prime}(x)\right|$

At last, for the general continuous approximated function $f(x)$, the following theorem of convergence is valid:

Theorem 3: If $f(x)$ is continuous in $[a, b]$, and the interpolation knots are $\left\{x_{j}=x_{0}+j h\right\}_{j=0}^{N}$ (namely equally distributed), then we have the estimation:

$$
\left\|f-L_{D} f\right\|_{\infty} \leq(1+2 c / h) \omega(f, h) .
$$

Proof: Due to

$$
\begin{aligned}
\mid L_{B} f-L_{D} f & |\leq| \frac{f\left(x_{1}\right)-f\left(x_{0}\right)}{x_{1}-x_{0}}\left\|\gamma_{0}(x)|+| \frac{f\left(x_{N}\right)-f\left(x_{N-1}\right)}{x_{N}-x_{N-1}}\right\| \gamma_{N}(x) \mid \\
& \leq \frac{\omega(f, h)}{h} c,
\end{aligned}
$$

using Theorem B, we have

$$
\begin{aligned}
\left\|f-L_{D} f\right\|_{\infty} & \leq\left\|f-L_{B} f\right\|_{\infty}+\left\|L_{B} f-L_{D} f\right\|_{\infty} \\
& \leq(1+c / h) \omega(f, h)+(c / h) \omega(f, h) \\
& =(1+2 c / h) \omega(f, h) .
\end{aligned}
$$


Citation: Zhang Y, Liang XZ, Li Q (2013) A Further Research on the Convergence of Wu-Schaback's Multi-quadric Quasi-Interpolation J Appl Computat Math 2: 138. doi:10.4172/2168-9679.1000138

Page 3 of 3

Remark 3: Assuming $c=O(h)$ in Theorem 3, we can conclude the convergence of Wu-Schaback's quasi-interpolation operator dealing with continuous approximated functions when the interpolated knots are equally distributed.

\section{Acknowledgement}

Supported by the National Natural Science Foundation of China (No.11271041, No.61170005) and the Natural Science Foundation of Jilin Province (No.20130101062JC).

\section{References}

1. Hardy RL (1971) Multiquadric Equations of Topography and Other Irregular Surface. J Geophysical Res 76: 1905-1915.

2. Franke R (1982) Scattered Data Interpolation: Tests of Some Methods. Math Comp 38: 181-200.
3. Powell MJD (1990) Univariate multiquadric approximation: reproduction of linear polynomials. In: Multivariate Approximation and Interpolation Basel: Birkhauser Verlag 227-240.

4. Beatson RK, Powell MJD (1992) Univariate Multiquadri Approximation: Quasiinterpolation to Scattered Data. Constr Approx 8: 275-288.

5. Beatson RK, Dyn N (1996) Multiquadric B-splines. Journal of Approximation Theory 87: 1-24.

6. Wu ZM, Schaback R (1994) Shape Preserving Properties and Convergence of Univariate Multiquadric Quasi-interpolation. Acta Math Appl Sinica (Engl Ser) 10: 441-446.

7. Zhang WX, Wu ZM (2004) Some Shape-preserving Quasi-interpolants to Nonuniformly Distributed Data by MQ-B-Splines. Appl Math J Chinese Univ Ser B 19: 191-202.

8. Ma LM, Wu ZM (2009) Approximation to the k-th Derivatives by Multiquadric Quasi-interpolation method. Journal of Computational and Applied Mathematics 231: 925-932. 